\title{
Digitale Ungleichheiten und digitale Spaltung
}

\author{
Norbert Kersting
}

\section{Inhalt}

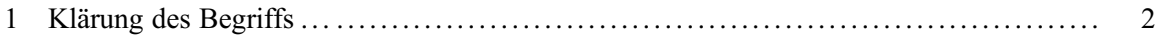

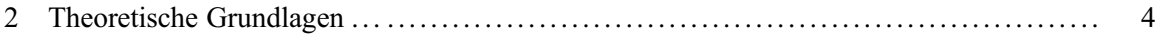

3 Ausmaß und Struktur digitaler Ungleichheiten: Untersuchungsergebnisse ............ 5

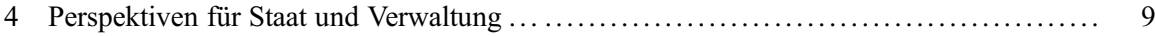

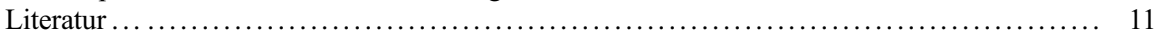

\section{Zusammenfassung}

Als digitale Spaltung werden ungleiche individuelle und gruppenspezifische Zugangschancen zu digitalen Informations- und Kommunikationstechnologien verstanden. Digitale Spaltung umfasst neben internationalen Unterschieden bezüglich des Digitalisierungsniveau auch regionale Ungleichheiten. Der Zugang zu digitaler Infrastruktur ist in Deutschland zum Teil noch rückständig, was sowohl temporär (bei stärkerer Nutzung) als auch regional (auf dem Land) deutlich wird. Neben den Zugangsmöglichkeiten als erstem Level der digitalen Spaltung sind als zweites Level die Kenntnisse und Kompetenzen der Bürger*innen relevant. Diesbezügliche Disparitäten zeigen sich beim Vergleich zwischen Bevölkerungsgruppen wie Jugendlichen und Senioren, Männern und Frauen (Gender Gap), aber auch bei marginalisierter armer Bevölkerung, Gruppen mit Migrationshintergrund oder Menschen mit Behinderungen. Digitale Spaltung überlappt und verstärkt somit bestehende soziale und partizipative Ungleichheiten. Lösungsvorschläge stellen neben der Anerkennung der digitalen Infrastrukturzugänge als Teil staatlicher Daseinsvorsorge die digitale Gleichheit (Netzneutralität) in den Vordergrund. Zum anderen sollen gemischte (blended) Partizipations- und Bildungsangebote die notwendigen Kennnisse vermitteln.

\footnotetext{
N. Kersting $(\bowtie)$

Institut für Politikwissenschaft, Universität Münster, Münster, Deutschland

E-Mail: norbert.kersting@uni-muenster.de
} 


\section{Schlüsselwörter}

Digitale Infrastruktur · Netzneutralität · Medienkompetenzen · Gender Gap · Politische Partizipation

\section{$1 \quad$ Klärung des Begriffs}

Mit dem Begriff digitale Spaltung werden die ungleichen Zugangsmöglichkeiten zu den digitalen Informations- und Kommunikationstechnologien beschrieben. Bevölkerungsgruppen können von deren Nutzung aufgrund mangelnder Infrastruktur und/oder fehlender individueller Ressourcen ausgeschlossen sein. Insofern wird auf einer ersten Ebene digitaler Spaltung (First Level Digital Divide, Makroebene) als mangelhafte Versorgung mit digitaler Infrastruktur (Norris 2001) und auf einer zweiten Ebene (Second Level Digital Divide, Mikroebene) als unterschiedlich ausgeprägte Medienkompetenzen definiert (Mossberger et al. 2008).

First Level: Digitalisierung bewirkt über eine Ausweitung der Nutzer*innen als auch über größere Datenmengen neuer Onlinedienste höhere Ansprüche an die Infrastruktur. Aufgrund der starken Visualisierung von neuen Dienstleistungsplattform im Internet wie zum Beispiel YouTube, Netflix und Zoom sind größere und schnellere Datentransfers notwendig. Von zentraler Bedeutung ist dabei neben dem Durchfluss die Geschwindigkeit innerhalb der digitalen Infrastruktur. Bei starker Nutzung (wie z. B. der Covid-19-Pandemie) sind die Netze überlastet. Dabei wird in Deutschland flächendeckend eine mangelnde Versorgung der Haushalte mit schnellen Glasfaserzugängen deutlich, die zudem den ländlichen Raum ausgrenzt. Man kann neben der nationalen auch eine internationale Ungleichheit in Bezug auf die Digitalisierung feststellen. Auf der einen Seite zeigen sich im globalen Vergleich Länder, in denen das Millennium Development Goal No. 8 zur Digitalisierung kaum oder nicht erreicht wurde und ein Großteil der Bevölkerung von diesen digitalen Netzen und Serviceleistungen ausgeschlossen ist (UN 2015, S. 83). Dabei wird bei den Indikatoren zwischen der Nutzung von Mobiltelefonen, Personal Computern sowie anderen Internet-Zugängen unterschieden. Hier wird eine führende Rolle der OECD-Länder konstatiert. Während traditionelle Informationstechnologien wie zum Beispiel Telefonleitungen hier deutlich weiterverbreitet sind als zum Beispiel in Asien, Lateinamerika oder Afrika, so galt dies auch für die Nutzung von Computern sowie von Internetanschlüssen. In Europa liegen dabei die skandinavischen Länder vor Mitteleuropa und diese wiederum vor den südlichen Ländern Europas. Gleiches galt für die Nutzung für Mobiltelefone, bei denen die Länder des Südens jedoch deutlich aufholten. In den Jahren seit 2000 zeigt sich dabei eine Tendenz, dass nicht allein die Verbreitung von Personal Computern ausschlaggebend ist. Aufgrund der weiten Verbreitung von Mobiltelefonen wurde insbesondere in einigen ostafrikanischen Ländern wie zum Beispiel Ruanda und Kenia ein Schwerpunkt auf die Entwicklung von mobilen Technologien gesetzt („,mobile first“). Insofern wird Afrika der erste Post-PC-Kontinent sein, in denen wesentliche digitale Dienstleistungen über Mobiltelefone umgesetzt werden. Afrika ist in Bezug auf die Ein- 
führung von mobilem E-Banking auch den OECD-Ländern deutlich voraus. In Europa und den USA wurde die Mobile-First-Strategie erst deutlich später aufgegriffen.

Second Level: Individuelle Ressourcen wie z. B. Medienkompetenzen umfassen digitale Kenntnisse, die das Mediennutzungsverhalten insbesondere bei politischer Beteiligung prägen. In Zusammenhang mit gesellschaftlicher Ungleichheit und sozialer Spaltung werden divergierende kognitive Ressourcen auch in Bezug auf die Kenntnisse der digitalen Medien deutlich (van Dijk 2005). So unterscheiden sich diese Kenntnisse über innovative digitale Technologien in verschiedenen Milieus, in sozialen Gruppen wie auch in den unterschiedlichen Alterskohorten. Insofern gibt es eine Überlappung von sozialer (arm vs. reich), partizipativer (aktiv vs. passiv) und digitaler Spaltung. Deutschland liegt dabei in Bezug auf die Nutzung der sozialen Medien deutlich hinter anderen westlichen Industrienationen wie Frankreich und den USA zurück. Dennoch zeigt sich auch hier eine zunehmende Zahl deutscher Nutzer (ARD-ZDF 2017). Im deutschen Kontext wird deutlich, dass sich aber insbesondere die Multiplikatoren im politischen Bereich, wie zum Beispiel Journalisten und Politiker, in Deutschland stärker in den sozialen Medien wie zum Beispiel Twitter engagieren. 2016 besaßen ca. 40 Millionen Deutsche ein Smartphone. 33 Million Nutzern greifen auf Messengerdienste wie zum Beispiel WhatsApp zurück. Es existierten 24 Millionen Facebook-Accounts, aber nur etwa 4 Millionen Twitter-Accounts. Hierbei lässt sich jedoch nur begrenzt von der Anzahl der Accounts auf die möglichen Nutzungsmuster schließen, da viele dieser Accounts lediglich sporadisch oder gar nicht benutzt werden. So ist zu beachten, dass man entsprechend der 90-9-1 Regel davon ausgehen kann, dass mehr als $90 \%$ aller Nutzer lediglich Informationen im Netz beobachten (Lurkers). Demgegenüber stehen etwa $9 \%$, die die neuen Medien sporadisch aktiv benutzen und etwa 1 Prozent starke Nutzer (Super Twitteroos) (Roemmele 2012).

Die internationalen Entwicklungstrends orientieren sich weg vom PC hin zu mobilen Endgeräten wie zum Beispiel Smartphones, wobei noch Generationsunterschiede deutlich werden. Das Smartphone wird in Deutschland durchschnittlich täglich bis zu 89 Mal benutzt (Initiative D21 2018). Während die Babyboomer Generation (bis 1965) als digitale Immigranten sich erst in der Postadoleszenz mit der Digitalisierung befasste, sind die jüngeren Generationen deutlich früher hiervon betroffen. Die Generation X (bis 1980) hat bereits die Entwicklung der sozialen Medien in der Adoleszenz wahrgenommen. Dennoch ist diese Generation häufig auch aufgrund stark materialistischer Ausrichtung - eher durch eine geringere politische Beteiligung charakterisiert. Waren lange Zeit die mittleren Bevölkerungsgruppen im Alter zwischen 30 und 50 Jahren die aktiven Träger sowohl des politischen als auch des sozialen Engagements, sind diese zunehmend durch ökonomische Bindungen und stärker familienorientierte Aktivitäten weniger politisch aktiv als noch in den davorliegenden Jahrzehnten (Kersting 2016a). Beim digitalen politischen Engagement werden sie mittlerweile bei den Wachstumszahlen von den Babyboomern und jungen Senioren übertroffen. Dieses geringere politische Engagement im Alter von 25-55 Jahren galt lange Zeit als Phänomen, das insbesondere 
Frauen betraf, die im mittleren Alter vor allem mit der Kindererziehung stark beschäftigt waren. Mittlerweile zeigt sich bei beiden Geschlechtern in dieser Altersgruppe eine deutliche Abnahme politischen Engagements (Kersting 2016b), während die jüngeren Generationen sich weiterhin stark engagieren. Diese Generation X (1965-1980) ist zwar internetaffin, aber beruflich stark eingebunden, während die Generation Y (1990-2000) und die Millennials (seit 2000) bereits als Digital Natives gelten. Mit der Weiterentwicklung der Digitalisierung und der Dominanz des Smartphones sind sie bereits im vorpubertären Kindesalter digital sozialisiert.

\section{Theoretische Grundlagen}

Im Folgenden wird digitale Spaltung in Bezug auf politische Online-Partizipation und die Nutzung staatliche Dienstleistungen analysiert. Digitale Aktivitäten sind nur sehr begrenzt politisches Engagement. Die Mehrzahl der Nutzung digitaler Medien bezieht sich auf den ökonomischen und den Unterhaltungsbereich. Internetnutzer werden als Shopper, Chatter oder Gamer tituliert. Die Mehrzahl der Nutzung ist freizeitorientiert und unterhaltungsorientiert. Politische Online-Partizipation und Online-Verwaltungskontakte sind eher seltener.

Die Gateway-These behauptete daher ein wachsendes Engagement vormals inaktiver Bürger. Die Substitutionsthese sah dagegen in der Digitalisierung lediglich neue Kanäle für ohnehin bereits politisch Aktive. Hierdurch könnte sich die partizipative Spaltung eher vergrößern als verkleinern. Die übertriebenen Erwartungen der Vertreter der Gateway-These wurden vielfach enttäuscht, da politisches Engagement eher über Themen als über Kanäle forciert wird. Dennoch war die digitale Mobilisierungsfunktion wichtig. Die Substitutionsthese übersah, dass insbesondere die jüngeren Generationen der Millennials ohnehin digital sozialisiert wurde. Häufig sieht man bei den Digital Natives ein zweites, virtuelles Leben im Internet. Erste digitale politische Erfahrungen tragen zu späterer analoger Offline-Partizipation bei.

Bei der Analyse der gesellschaftlichen Gruppen, des politischen Engagements sowie der Nutzung von digitalen Medien lässt sich neben einer digitalen Spaltung auch eine soziale und eine partizipative Spaltung darstellen. Die soziale Spaltung basiert auf ungleichen Teilhabechancen von verschiedenen Gruppen definiert nach Einkommen, Geschlecht, Alter und weiteren sozialen Charakteristika wie z. B. Migrationshintergrund. Die partizipative Spaltung unterscheidet politisch aktive und inaktive Gruppen. Letztere lassen sich in ,politische Zyniker“ und ,politisch apathische Bürger" unterteilen (Kersting 2013). In der ersten Dekade des neuen Millenniums zeigte sich zunächst eine Reaktivierung der zynischen politischen Gruppen. Diese besitzen relativ hohe politische Kenntnisse, sehen aber ein Engagement im politischen System als nicht effektiv an. Aufgrund dieser geringen politischen externen Efficacy, also dem mangelnden Vertrauen in politische Einflussmöglichkeiten, engagierten sich diese Gruppen lange Zeit nicht. Mit der Protestwelle um Stuttgart 21 und einem neuen Engagement dieser häufig aus der BabyboomerGeneration rekrutierten Bevölkerungsgruppe kam es seit den 2010er-Jahren zu einer Re-Aktivierung dieser Gruppe als „Wutbürger“. Im Rahmen der Kritik an dem 
politischen Establishment und einer neuen, insbesondere rechtspopulistischen Welle, zeigen sich zudem eine neue Aktivierung von Bevölkerungsgruppen, die lange Zeit eher als politisch apathisch angesehen wurden. Diese attestierten sich häufig eine geringe interne Efficacy, das heißt, sie sahen sich als nicht kompetent in Bezug auf politisches Engagement an. In dieser Gruppe kam es durch rechtspopulistischen Protest und durch die Entwicklung neuer politischer Parteien zu einer Reaktivierung.

Die Digitalisierung wurde lange Zeit als große Chance zur politischen Mobilisierung über Onlinekanäle angesehen (Norris 2001). Neue Öffentlichkeiten sollten die demokratischen Systeme revitalisieren. Dabei spielt Online-Partizipation eine besondere Rolle in der politischen Sozialisation von Jugendlichen, da sie den Einstieg in das politische Engagement beinhalten konnte. Online Medien bewirken danach eine Mobilisierung und hieraus resultierend auch Offline-Engagement. Über Online-Partizipation in sozialen Netzwerken (Facebook, Twitter, Instagram) lassen sich neben dialogischen Instrumenten auch stark demonstrative Partizipationsformen realisieren. In diesem Zusammenhang ist die Entwicklung von Echokammern und Teil-Öffentlichkeiten, d. h. segmentierten Diskursarenen im Internet, von besonderer Bedeutung. Hier können auf der einen Seite die individuelle Identitätsfindung in einem etwas breiteren gesellschaftlichen Diskurs bewerkstelligt werden und dabei die Entwicklung von neuen politischen Gemeinschaften vorangetrieben werden. Problematisch ist aber, dass in diesem Bereich eine besondere Konzentration auf partikulare Einzelinteressen, eine geringe Reflexivität, eine Radikalisierung und oft eine stärkere Ausländerfeindlichkeit deutlich wird.

\section{Ausmaß und Struktur digitaler Ungleichheiten: Untersuchungsergebnisse}

Im Folgenden wird zunächst die digitale Infrastruktur als erste Ebene der Digitalen Spaltung analysiert, bevor divergierende Kompetenzen verschiedene Bevölkerungsgruppen untersucht werden. Bei der Betrachtung unterschiedlicher sozialer Gruppen zeigen sich deutliche Ausgrenzungstendenzen und mangelnde digitale Inklusion. In den weiteren Abschnitten werden die Gruppen Jugendliche, Senioren, Menschen mit Migrationshintergrund und Menschen mit Behinderung näher analysiert. Weiterführende Analysen zu Gruppen wie z. B. prekäre oder arme Haushalte fehlen bislang.

\subsection{Zugangsmöglichkeiten und digitale Infrastruktur}

Der Breitbandatlas der Initiative D 21 sieht 2011 grundsätzlich zwei Drittel der Bevölkerung mit einem Zugang von mehr als $16 \mathrm{MBit} / \mathrm{s}$. Bezüglich der Breitbandverfügbarkeit der Bundesländer wurden bereits damals Disparitäten deutlich. 2017 sind demnach nahezu alle Haushalte mit einer Geschwindigkeit von mindestens $6 \mathrm{MBit} / \mathrm{s}$ abgedeckt. Es zeigen sich ab einer Größe von mehr als $30 \mathrm{MBit} / \mathrm{s}$ regionale 
Disparitäten (BMVI 2017). Während Länder wie Bayern und Baden-Württemberg über $84 \%$ und in den Stadtstaaten sogar über $95 \%$ mit dieser schnellen Technologie versorgt werden, liegen Länder wie zum Beispiel Mecklenburg-Vorpommern (67 \%) oder Brandenburg (71 \%), Sachsen (69 \%) und Sachsen-Anhalt (60 \%) zurück. Es zeigt sich somit eine Ungleichverteilung zwischen neuen und alten Bundesländern. Zudem sind diese Geschwindigkeiten nicht in Spitzenzeiten bei einer hohen Anzahl von Nutzern zu erreichen, da es sich zumeist nicht um Glasfasertechnologien handelt. Auch bei der Mobilfunk LTE-Versorgung wird davon ausgegangen, dass grundsätzlich mehr als $96 \%$ der Haushalte hiermit versorgt sind (BMVI 2017). Doch auch hier besteht in den strukturschwachen ländlichen Regionen sowie bei hoher Nutzung eine deutlich geringere Verfügbarkeit. Die Bandbreitenklassen werden weiterhin insbesondere durch DSL bzw. VDSL abgedeckt, $d$. h. es werden nur die Stadtteile und nicht die Haushalte über Glasfaseranschlüsse verbunden. Weitere Anbieter wie Kabel Deutschland oder Unitymedia besitzen deutlich geringere Geschwindigkeiten. Von besonderer Bedeutung ist auch die gewerbliche Breitbandbzw. Glasfaser-Verfügbarkeit. Diese liegt bei den Leitungen mit mehr als $50 \mathrm{Mbit} / \mathrm{s}$ bei allen Firmen in Gewerbegebieten deutlich höher (ca. 80 \%), während Mischgebiete nur ca. $70 \%$ dieser Geschwindigkeit erreichen. Obwohl diese regionalen Ungleichheiten beim Netzausbau existieren, gibt es bei der Infrastruktur keine Diskriminierung von Gruppen. Die Netze selbst erzeugen keine Bevorzugung einzelner Gruppen. Bislang besteht eine hohe Netzneutralität, das heißt es werden grundsätzlich keine Arten von Datenübertragungen im Internet bevorzugt oder benachteiligt. Neben der Geschwindigkeit der digitalen Infrastruktur in Bezug auf den Datendurchsatz ist die Latenz (Reaktionszeit) von besonderer Bedeutung. Hiervon hängen neue Möglichkeiten für Technologien wie zum Beispiel autonomes Fahren ab.

\subsection{Jugendliche}

Die jüngere Generation galt lange Zeit als politisch desinteressierte, beteiligungsferne und schwer erreichbare Gruppe, die aber stark durch die Digitalisierung beeinflusst werden kann. Insofern wurde der Digitalisierung und den neuen Kanälen im Internet eine besondere Rolle bei der Sozialisation zugewiesen. Über neue Informations- und Kommunikationstechnologien sollten insbesondere Jugendliche stärker an die Politik gebunden werden und mobilisiert werden. Auch die Angebote der kommunalen Verwaltungen sollten - so die Annahme - von dieser Gruppe stärker genutzt werden. Ein mangelndes politisches Engagement kann laut neuerer Untersuchungen wie zum Beispiel der vorletzten Shell Jugend-Studie (2015) nicht mehr festgestellt werden. Gleichzeitig wurde der Generation Y und den Millennials ein größeres politisches Online-Engagement attestiert. Die Jugendlichen unterscheiden sich diesbezüglich beim politischen Engagement kaum von älteren Kohorten wie den Babyboomern. Es wird allerdings deutlich, dass es eine geringere feste Zuordnung zu politischen Parteien und zum sozialen Organisationen wie Kirchen gibt, die ein regelmäßiges Engagement beinhaltet. Jugendliche wie andere soziale Gruppen 
binden sich zunehmend nur sporadisch an neue soziale Bewegungen und Organisationen. Ihr Engagement bleibt stark auf einmalige „Events“ bezogen und ist auch aufgrund der Suche nach der eigenen Identität in deutlich höherem Maße expressiv (Kersting 2016b).

\subsection{Senioren und Silver Surfer}

In den 1980ern wurde Senioren im Pensionsalter ab 65 häufig ein politisches Disengagement attestiert. Auch die Internetnutzung in dieser Altersgruppe lag am Ende der 1990er lange Zeit deutlich zurück (Statistisches Bundesamt 2016, S. 78 ff.). Entsprechend damaliger (Dis-) Engagement-Theorien nahm nach dem 65. Lebensjahr das politische Engagement aufgrund mangelnder Ressourcen und vor allem auch aufgrund eines schlechteren Gesundheitszustandes ab. Dies hing - insbesondere in den älteren ärmeren Bevölkerungsgruppen in den ländlichen Regionen - mit einem niedrigeren Bildungsstand und mangelnden politischen Kenntnissen zusammen. Bei der Babyboomer-Generation zeigt sich dagegen bereits eine starke Politisierung, die mit einer erhöhten Lebenserwartung und grundsätzlich höherem Engagement im Alter korreliert. Ein hohes politisches Engagement - insbesondere gegen infrastrukturelle Großprojekte wie zum Beispiel Stuttgart 21 - hat zur Mobilisierung von älteren etablierten Mittelschichten führt. Die Sitzblockade war nicht nur in der Mitte der Gesellschaft angekommen, sondern auch bei den Senioren. Senioren besitzen die für Partizipation notwendigen Zeitressourcen wie auch die kognitiven Ressourcen und oft auch die ökonomische Unabhängigkeit, um sich politisch stärker einzubringen. Dabei zeigt sich im linken Spektrum eine Reaktivierung der „politischen Zyniker“. Zudem wird auch in den letzten Jahren ein starker Protest im Bereich von migrationsfeindlichen rechtspopulistischen Gruppen deutlich. Hier existieren starke Anti-Establishment-Einstellungen. Befürchtungen eines Zerfalls der nationalen Identität und eines drohenden sozialen Abstiegs gelten als Ursachen für politisches Engagement.

In dieser älteren Altersgruppe zeigen sich aber in den letzten Jahren gravierende Veränderungen bei der Online-Partizipation. Dabei liegen die Ursachen insbesondere in zwei Faktoren. Auf der einen Seite ist die Kohorte der Babyboomer weitgehend in das Rentenalter aufgerückt und anderseits sind die technischen Möglichkeiten erweitert worden und somit Barrieren des digitalen Engagements für Ältere deutlich gesunken. Neuere Laptops und bedienungsfreundliche Tablets sind auf dem Markt. Dabei zeigte sich ein drastischer Anstieg der Internetnutzung. Neuere Zahlen zeigen, dass zwei Drittel der Nutzer der älteren Generation regelmäßig im Internet und zum Teil täglich online aktiv sind (Kersting 2015). Ein starker Fokus liegt auf Informationsbeschaffung über Online-Angebote der großen Zeitungen und Zeitschriften, die von zwei Drittel der Nutzer frequentiert werden. Weniger als ein Drittel sind in sozialen Netzwerken wie zum Beispiel Facebook aktiv. Der Fokus liegt insbesondere auf sozialen und ökonomischen Aktivitäten und im Rahmen der Kommunikation mit den Familienmitgliedern vor allem auf Messengerdiensten wie zum Beispiel WhatsApp. Bisher unterschiedliche digitale Nutzungsverhalten glei- 
chen sich heute langsam an, da bereits jetzt so genannten Silver Surfer die neue Generation der Senioren darstellen und diese sich weniger über Ressourcenmangel (Zeit, Einkommen, Kenntnisse zu Digitalisierung, Computerkenntnisse etc.) charakterisieren. Erst im sehr hohen Alter beginnt das Disengagement und eine deutlich geringere Nutzung unterschiedlicher Kommunikationskanäle.

\subsection{Digitale Geschlechterungleichheit und der Gender Gap}

Die digitale Spaltung spiegelt sich auch in der Geschlechterungleichheit in verschiedenen Bereichen wider. Frauen finden sich nicht nur seltener in den Führungsetagen der großen digitalen Konzerne. Sie sind außerdem in der Gruppe der Softwareentwickler unterrepräsentiert. Dies scheint damit zusammenzuhängen, dass die Technikaffinität bei vielen Frauen bislang weniger stark ausgeprägt ist als bei Männern (Langer 2012). Dabei zeigen sich sowohl geschlechtsbezogen unterschiedliches Nutzungsverhalten als auch Tendenzen einer Nivellierung dieser Unterschiede. Deutlich wird, dass grundsätzlich Frauen weniger Zeit zum Surfen und zum Downloaden aufwenden. Sie nutzen deutlich mehr Zeit für Informationsseiten und Diskussionsseiten im Bereich Kultur und Freizeit. Männer nutzten das Internet hingegen vermehrt für Video-Konsum. Dies gilt insbesondere für soziale Medien wie zum Beispiel YouTube. Gleichzeitig waren sie stärker in Weblogs und bei Kommentaren im Internet aktiv. Bei den Senioren sind Unterschiede zwischen Männern und Frauen offensichtlich. Während etwa drei Viertel der Männer zwischen 65 und 75 täglich aktiv surfen, sind dies nur etwas mehr als die Hälfte der Frauen. Auch bei Internetnutzern, die für die Entwicklung neuer Software eine besondere Rolle spielen, sind Frauen eher unterrepräsentiert. Typische Internetpioniere und First User sind männlich, zwischen 20 und 40 Jahre alt, beschäftigt, hochgebildet und nutzen das Internet sowohl für ökonomische Zwecke als auch zur Unterhaltung.

\subsection{Digitale Integration und Migration}

Bevölkerungsgruppen mit Migrationshintergrund sind häufig sozial marginalisiert, aber auch Ziel diverser Integrationspolitiken. Dies macht sich besonders deutlich in der Gruppe der Flüchtlinge. Personen mit Migrationshintergrund und insbesondere Flüchtlinge besitzen in der Regel Mobiltelefone zur internen Kommunikation und als Medium der Information über das Heimatland. Während Flüchtlinge durch die Nutzung von Messengerdiensten und E-Mail sowohl den Kontakt zu ihren Familien in den Kriegs- und Krisenregionen herstellen, sind sie von der Nutzung digitaler Angebote in Deutschland oft bereits sprachlich ausgeschlossen. Die Internetseiten von deutschen Behörden sind vielfach nicht auf Englisch oder in anderen Sprachen verfügbar. Sie verwenden oft keine leicht verständliche Sprache. Weiterhin sind Bereiche wie E-Learning und Plattformen für den Spracherwerb kaum vorhanden bzw. oft nicht auf mobile Endgeräte zugeschnitten. 


\subsection{Behinderung und Exklusion}

Die Gruppe der Menschen mit Behinderungen in Deutschland ist durch eine starke Heterogenität gekennzeichnet. Darauf reagiert ein breites Angebot im Bereich der Information und des Lernens. Für diese Bevölkerungsgruppe ist Barrierefreiheit von zentraler Bedeutung. Die Internetseiten von staatlichen Behörden auf lokaler, regionaler und nationaler Ebene als auch zivilgesellschaftliche und privatwirtschaftliche Angebote weisen eine derartige Barrierefreiheit und Formulierungen in leichter Sprache allerdings nur selten auf (Wei und Hindman 2011).

\section{$4 \quad$ Perspektiven für Staat und Verwaltung}

Die Strategien zur Auflösung der digitalen Spaltung auf der infrastrukturellen Ebene (First Level Digital Divide auf der Makroebene) und auf der individuellen Ressourceneben (Second Level Digital Divide auf der Mikroebene) sind vielfältig. Auf dem ersten Level der infrastrukturellen Zugangsmöglichkeiten liegt der Hauptbedarf und eine wichtige Aufgabe in der Bereitstellung dieser Infrastruktur für die einzelnen Haushalte. Insofern geht es um den Einsatz von Glasfasertechnologie oder ähnlich schneller Infrastruktur in allen Regionen. Hierfür muss die digitale Infrastruktur als Teil der Daseinsvorsorge anerkannt werden. Digitale Bürgerrechte und digitale Souveränität können nur über das Recht auf gleiche Zugangsmöglichkeiten zu den digitalen Angeboten realisiert werden. Aufgrund vielfachen Marktversagens scheinen die notwendigen breiten Zugangsmöglichkeiten nur über eine staatliche Bereitstellung der Netzinfrastruktur bewerkstelligt werden zu können. In Deutschland hatten 2017 nahezu alle Bevölkerungsgruppen einen schnellen Internetzugang mit max. 25 Mbit/Sekunde (BMVI 2017). Dies gilt aber nicht in Spitzenzeiten und auch nicht flächendeckend. Es zeigen sich leichte Unterschiede bei den Bundesländern (Ost-West) und zwischen ländlichen und städtischen Regionen. Diese aggregierten Zahlen sind zum Teil irreführend, da diese Geschwindigkeit in Spitzenzeiten real nicht erreicht werden können. Bei stärkerer Nutzung und insbesondere in Krisenzeiten wie bei der Covid-19 Pandemie sind die Netze überlastet. Hier ist die Nutzung von Breitbandtechnologien, die auf Glasfaservernetzung bei den Hausanschlüssen setzen, der zentrale Indikator, da diese einen hohen Datentransfer erlaubt. Die schnellen Glasfasertechnologien können bislang nur von wenigen Bevölkerungsgruppen genutzt werden. Seit 2017 setzt man flächendeckend auf diese Technologie und verzichtet auf den Ausbau von anderen, deutlich langsameren und begrenzten Zwischentechnologien.

Ältere internationale Lösungswege wie z. B. in Griechenland sahen unter anderem Telezentren, Multi-Zweck-Gemeinschaftszentren und Terminals in öffentlichen Gebäuden wie zum Beispiel in Büchereien oder Poststation vor. Mittlerweile wird diese Idee der digitalen Gemeinschaftserlebnisse wieder aufgegriffen. So bieten Vereine digitale Abteilungen im E-Sport an, in denen vor allem Jugendliche gemeinsam elektronische Sportarten praktizieren können. Gleichzeitig gründen sich vereinzelt multikulturelle Computer-Clubs, die zur Integration und indirekt zur Ausbildung 
von Jugendlichen zumeist mit Migrationshintergrund beitragen. Die gemeinsame Nutzung von digitalen Infrastrukturen in Nachbarschaftszentren wie zum Beispiel Bibliotheken, aber auch digitale Plattformen (nebenan.de) dienen auch der dient auch der Entwicklung von analogen nachbarschaftlichen Netzwerken und Gemeinschaft.

Digitalisierung hat zu einer verstärkten Individualisierung beigetragen. Digitale Enklaven und Informationsblasen in den sozialen Medien, die sich oft durch Dominanz von Hate Speech auszeichnen, prägen den sozialen wie politischen Diskurs. Die Aufgabe von politischen Verwaltungen und Parteien und anderen staatlichen wie zivilgesellschaftlichen Institutionen wird es zunehmend sein, diese segmentierten Arenen zu einer gemeinsamen Diskursarena zusammenzufügen, um ein gegenseitiges Verstehen zu ermöglichen. Der Abbau von digitaler Spaltung kann dabei zu Integration und Inklusion beitragen.

Dabei ist die Entwicklung von Instrumenten der Bürgerbeteiligung, die OnlinePartizipation und face-to-face-Kommunikationen miteinander verbinden (blended participation), von besonderer Bedeutung. Hierüber könnten besondere Medienkompetenzen vermittelt werden, die zu neuen Digital Skills, aber auch zu einem neuen medialen Habitus und zur Netiquette beitragen. Die Nutzung von digitalen Technologien zur Vernetzung von Bürgern auf der lokalen Ebene, in der Nachbarschaft und in der Stadt muss im Rahmen einer Smart City einen hohen politischen Stellenwert besitzen. Hierüber lassen sich nicht nur neue nachhaltige Strategien und Plattformen eine Sharing Economy entwickeln, sondern auch neue Gemeinschaften, die zentrale Aufgaben im neuen Millennium wie zum Beispiel Seniorenbetreuung und Integration aufgreifen können.

Bei Jugendlichen ist sozialer Protest Teil der Identitätsfindung und der Jugendkultur. Neue Online- und Offline-Partizipationsangebote (invited space) der Politik und Verwaltung, der soziale Bewegungen und der politischen Parteien können für Jugendliche attraktiv sein, wenn auch Bedürfnisse nach Expressivität und Identitätssuche beachtet und einbezogen werden. Dabei drängen Jugendliche auf eine Entwicklung selbstbestimmter, relativ autonomer Räume (invented space), um insbesondere Kontrollmechanismen und der Dominanz durch Hierarchien zu entfliehen. Insofern wird eine zu deutliche Hinwendung der Politik und Verwaltung an vermeintliche Bedürfnisse von Jugendlichen von diesen selbst als Anbiederung wahrgenommen und führt zu einer Gegenbewegung und Gegenöffentlichkeit, um sich gerade von diesem invited space abzugrenzen.

Multiple sozioökonomische und kulturelle Spaltungen der Gesellschaft wirken sich auch auf den Prozess der Digitalisierung der Gesellschaft aus. Zwar sind Strategien und Ansätze zur Auflösung speziell der digitalen Spaltung nötig. Eine langfristige Lösung der Probleme und ein gleichmäßiger digitaler Zugang können aber sicherlich nur über einen Abbau der sozioökonomischen Unterschiede erreicht werden. Der digitalen Spaltung auf der Makroebene als auch den mangelnden Medienkompetenzen auf der Mikroebene, die zu prekärer digitaler Souveränität beiträgt, kann zwar durch direkte Bildungsmaßnahmen entgegengewirkt werden, langfristig bedarf es aber auch einer Entwicklung in Richtung größerer sozioökonomischer Gleichheit. 


\section{Literatur}

ARD-ZDF. 2017. ARD/ZDF-Onlinestudie 2017. http://www.ard-zdf-onlinestudie.de/archiv-1997$2018 /$.

BMVI. 2017. Aktuelle Breitbandverfügbarkeit in Deutschland. Erhebung des TÜV Rheinland im Auftrag vom BMVI. Berlin: Bundesministerium für Verkehr und digitale Infrastruktur.

Initiative D21. 2018. D21 Digital index 2017/2018. Berlin: Initiative D21 e.V.

Kersting, Norbert. 2013. Online participation. From ,invited' to ,invented' spaces. International Journal of Electronic Governance 6(4): 270-280.

Kersting, Norbert. 2015. Senioren und politische Online-Partizipation. Engagement und Disengagement der Silversurfer. Zeitschrift für Politikberatung 7(4): 160-172.

Kersting, Norbert. 2016a. Politische Online-Beteiligung im internationalen Vergleich. Eine Revitalisierung politischer Beteiligung? Zeitschrift für Vergleichende Politikwissenschaft 10(2 Suppl): 91-113.

Kersting, Norbert. 2016b. Onlinebeteiligung und Jugendliche. Expressiv und unabhängig? Der Bürger im Staat 66(4): 278-285.

Langer, Christian. 2012. Digitale Spaltung. Eine kritische Analyse. Saarbrücken: Akademikerverlag.

Mossberger, Karen, Caroline J. Tolbert, und Ramona S. McNeal. 2008. Digital citizenship. The internet, society, and participation. Cambridge, MA/London: The MIT Press.

Norris, Pippa. 2001. Digital divide. Civic engagement, information poverty, and the internet worldwide. Cambridge: Cambridge University Press.

Roemmele, Andrea. 2012. Electronic political campaigning. In Electronic democracy, Hrsg. Norbert Kersting, 103-124. Opladen: Barbara Budrich.

Shell. 2015. Shell Jugend Studie 2015. https:/www.shell.de/ueber-uns/shell-jugendstudie.html\#va nity-aHR0cHM6Ly93d3cuc2hlbGwuZGUvanVnZW5kc3R1ZGll. Zugegriffen am 02.05.2020.

Statistisches Bundesamt. 2016. Ältere Menschen in Deutschland und der EU. Wiesbaden: Statistisches Bundesamt. https://www.destatis.de/Migration/DE/Publikationen/Thematisch/Bevoelke rung/Bevoelkerungsstand/BroschuereAeltereMenschen.html. Zugegriffen am 02.04.2020.

UN. 2015. Millenniums-Entwicklungsziele. Bericht 2015. New York: Vereinte Nationen.

van Dijk, Jan A. G. M. 2005. The deepening divide. Inequality in the information society. Thousand Oaks: Sage.

Wei, Lu, und Douglas B. Hindman. 2011. Does the digital divide matter more? Comparing the effects of new media and old media use on the education-based knowledge gap. Mass Communication and Society 14(2): 216-235.

van Dijk, Jan A. G. M. 2005. The deepening divide. Inequality in the information society. Thousand Oaks: Sage. 